\title{
BC200 LncRNA a potential predictive marker of poor prognosis in esophageal squamous cell carcinoma patients
}

This article was published in the following Dove Press journal:

OncoTargets and Therapy

I5 April 2016

Number of times this article has been viewed

\author{
Rui-Hua Zhao',* \\ Cai-hua Zhu ${ }^{2, *}$ \\ Xiang-Ke Li' \\ Wei $\mathrm{CaO}^{3}$ \\ Hong Zong' \\ Xin-Guang $\mathrm{CaO}^{2,4}$ \\ $\mathrm{Hai}-\mathrm{Yan} \mathrm{Hu}^{5}$
}

'Department of Oncology, The First Affiliated Hospital of Zhengzhou University, ${ }^{2}$ Department of General Surgery, The Affiliated Cancer Hospital of Zhengzhou University, ${ }^{3}$ Department of Translational Medicine Center, Zhengzhou Center Hospital, ${ }^{4}$ Department of Digestive Disease, The First Affiliated Hospital of Zhengzhou University, Zhengzhou, Henan, ${ }^{5}$ Oncology Department, Shanghai Jiao Tong University Affiliated Sixth People's Hospital, Shanghai, People's Republic of China

*These authors contributed equally to this work
Correspondence: Xin-Guang Cao Department of Digestive Disease, The First Affiliated Hospital of Zhengzhou University, I Jianshe Road, Zhengzhou, 450002, People's Republic of China

Tel +86 37l 66295532

Email xinguangcao@।26.com

Haiyan $\mathrm{Hu}$

Oncology Department, Shanghai Jiao Tong University Affiliated Sixth People's Hospital, No 600 Yishan Road Xuhui District, Shanghai City, 200233, People's Republic of China Email xuril 104@I63.com
Objective: To explore the expression and prognosis significance of $\mathrm{BC} 200$ in esophageal squamous cell carcinoma (ESCC) patients who received radical resection.

Methods: We used quantitative real-time polymerase chain reaction to detect the expression level of BC200 in cancer tissue and paired adjacent normal tissue samples from 70 ESCC patients who received radical surgical resection and analyzed the correlation of the relative expression level of BC200 with clinical-pathological features and prognosis.

Results: We found that the relative expression of BC200 was significantly higher in ESCC tissues compared with adjacent normal tissue samples $(P=0.023)$. But the expression of $\mathrm{BC} 200$ were not related to clinical-pathological features, such as age, TNM stages, and histological grade $(P>0.05)$. Kaplan-Meier analysis showed that high expression levels of BC200 were correlated with poor prognosis in ESCC patients. Patients with a high level of BC200 had a shorter disease-free survival and overall survival than those with low BC200 expression $(P=0.034$ and $P=0.031$, respectively). On multivariate analysis, the hazard ratio (HR) of $\mathrm{BC} 200$ expression was $2.17(95 \%$ confidence interval $[\mathrm{CI}]=1.12-4.19, P=0.022)$ for disease-free survival and 2.24 (95\% CI=1.12-4.49, $P=0.023$ ) for overall survival.

Conclusion: Our results indicate that high expression of $\mathrm{BC} 200$ reflects poor prognosis and could serve as a novel predictive marker for ESCC patients who received radical resection.

Keywords: BC200, esophageal squamous cell carcinoma, predictive marker, poor prognosis, long noncoding RNAs

\section{Introduction}

Esophageal squamous cell carcinoma (ESCC) is one of the most common and fatal malignancies in northern China, South Africa, Turkey, and Iran. ${ }^{1}$ Although great progress has been made in the diagnosis and treatment of ESCC in recent years, the 5 -year survival rate is still $<50 \%{ }^{2}$ The main reason is that it is difficult to diagnose ESCC at early stages of disease; most cases are diagnosed at the advanced stage..$^{3,4}$ Although many genetic and epigenetic changes are found to be correlated with ESCC, the underlying mechanisms of carcinogenesis remain poorly understood. Therefore, it is necessary to identify new molecular markers associated with the progression of this disease and to find novel treatment targets for ESCC.

Long noncoding RNAs (LncRNAs) are RNA molecules whose length is longer than 200 nucleotides and are not translated into proteins. Recent evidences show that LncRNAs play important roles in numerous biological processes, including $\mathrm{X}$-chromosome inactivation, genomic imprinting, chromatin modification, gene transcription, and splicing. ${ }^{5-8}$ Increasing evidence indicates that some LncRNAs are 
widely involved in the development and progression of ESCC. For example, a few studies showed that HOTAIR predicts negative prognosis and exhibits oncogenic activity in ESCC..$^{9,10}$ Xie et $\mathrm{al}^{11}$ found that SPRY4-IT1 was also upregulated in ESCC and associated with poor prognosis. Li et a ${ }^{12}$ demonstrated that the relative level of UCA1 was significantly higher in ESCC tissues compared to the adjacent nontumor tissues, and remarkably higher expression of UCA1 was found in esophageal cancer cell lines compared with the immortalized esophageal epithelial cell line. The ESCC patients with higher UCA1 expression had an advanced clinical stage and a poorer prognosis than those with lower expression. They also indicated that downregulation of UCA1 decrease cell proliferation, migration, and invasion ability. However, research of LncRNAs in ESCC remains largely unknown.

BC200 is a noncoding RNA of about 200 bp that is selectively expressed in the primate nervous system and has been implicated in the regulation of local synaptodendritic protein synthesis in neurons and might be involved in the process of Alzheimer disease. ${ }^{13}$ BC200 RNA does not normally express in nonneuronal somatic cells. However, a study has demonstrated that $\mathrm{BC} 200$ is deregulated under certain neoplastic conditions, and is expressed in carcinomas of the breast, cervix, esophagus, lung, ovary, parotid, and tongue, but not in corresponding normal tissues. ${ }^{14}$ The expression of BC200 RNA in nonneural tumors may indicate a functional interrelationship with induction and/or progression of such tumors. However, the role of BC200 in ESCC is still unclear. In this study, we investigated the correlations between the expression of $\mathrm{BC} 200$ and the clinical-pathological features and survival outcomes of patients who received radical resection of ESCC.

\section{Materials and methods Patients and tissue samples}

A total of 70 fresh cancer tissue and paired adjacent tissue samples were obtained from patients with ESCC, which were collected between August 2011 and May 2013. These patients underwent radical surgical resection without preoperative chemotherapy or radiotherapy at Henan Cancer Hospital and the First Hospital of Zhengzhou University in the People's Republic of China. After surgical removal, all samples were processed and immediately stored in RNAlater RNA stabilization reagent at $-80^{\circ} \mathrm{C}$ until RNA extraction. All the patients were pathologically confirmed to be ESCC and were histologically graded in accordance with the World Health Organization classification. Patients were staged according to the seventh version of the American Joint Committee on
Cancer staging system. The follow-up data were obtained by reviewing the outpatient charts and contacting the patients by telephone or email. The detailed clinical-pathological characteristics of the recruited patients are summarized in Table 1. All study subjects provided their written informed consents for the use of their tissue samples in the present study. This project was approved by the Clinical Research Ethics Committee of Henan Cancer Hospital and the First Hospital of Zhengzhou University.

\section{RNA isolation, reverse transcription, and quantitative PCR}

Isolation of cancerous and noncancerous tissue specimens total RNA was extracted using TRIzol reagent (Invitrogen, Carlsbad, CA, USA). The RNA was reverse transcribed into cDNA and quantified using the SYBR Green BC200 two-steps test Kits (Shanghai Novland Co., Shanghai, People's Republic of China) according to manufacturer's

Table I Clinicopathological characteristics of the 70 patients who received radical resection of ESCC

\begin{tabular}{|c|c|}
\hline Characteristic & $\mathbf{N}(\%)$ \\
\hline \multicolumn{2}{|l|}{ Sex } \\
\hline Male & $46(65.7 I)$ \\
\hline Female & $24(34.29)$ \\
\hline \multicolumn{2}{|l|}{ Age (years) } \\
\hline$\leq 60$ & $21(30.00)$ \\
\hline$>60$ & $49(70.00)$ \\
\hline \multicolumn{2}{|l|}{ pT stage } \\
\hline $\mathrm{TI}-2$ & $15(21.43)$ \\
\hline T3-4 & $55(78.57)$ \\
\hline \multicolumn{2}{|l|}{ pN stage } \\
\hline Negative & $38(54.29)$ \\
\hline Positive & $32(45.7 I)$ \\
\hline \multicolumn{2}{|c|}{ Histological grade } \\
\hline $\mathrm{I}-2$ & 31 (44.29) \\
\hline $3-4$ & $39(55.7 I)$ \\
\hline \multicolumn{2}{|l|}{ TNM stage } \\
\hline$|-| \mid$ & $45(64.29)$ \\
\hline III & $25(35.7 I)$ \\
\hline \multicolumn{2}{|c|}{ Cancer location } \\
\hline Upper/middle & $52(74.29)$ \\
\hline Lower & $18(25.7 \mid)$ \\
\hline \multicolumn{2}{|c|}{ Adjuvant chemotherapy and/or radiotherapy } \\
\hline Yes & $31(44.29)$ \\
\hline No & $39(55.7 \mathrm{I})$ \\
\hline \multicolumn{2}{|c|}{ BC200 expression } \\
\hline Low & $35(50.00)$ \\
\hline High & $35(50.00)$ \\
\hline \multicolumn{2}{|c|}{ Relapse or metastasis } \\
\hline Yes & $38(54.29)$ \\
\hline No & $32(45.7 I)$ \\
\hline \multicolumn{2}{|l|}{ Death } \\
\hline Yes & $34(48.57)$ \\
\hline No & $36(5 \mathrm{I} .43)$ \\
\hline
\end{tabular}


instructions and BC200 levels were quantified using the Applied Biosystems 7500 Fast Real-Time polymerase chain reaction (PCR) System. A $20 \mu \mathrm{L}$ reverse transcription (RT) reaction mix contained $2 \times \mathrm{RT}$ master mix $10 \mu \mathrm{L}$, RT enzyme mix $1 \mu \mathrm{L}, \mathrm{BC} 200(G A P D H)$ RT primer $1 \mu \mathrm{L}$, RNA $5 \mu \mathrm{L}$, and nuclease-free water $3 \mu \mathrm{L}$. The mix was incubated at $42^{\circ} \mathrm{C}$ for 15 minutes, $85^{\circ} \mathrm{C}$ for 15 seconds. A $20 \mu \mathrm{L}$ PCR reaction mix contained $2 \times \mathrm{PCR}$ master mix $10 \mu \mathrm{L}, \mathrm{BC} 200$ (GAPDH) primer $1 \mu \mathrm{L}$, cDNA product $2 \mu \mathrm{L}$, and nuclease-free water $7 \mu \mathrm{L}$. Total 40 cycles of amplification were performed, each cycle consisting of $94^{\circ} \mathrm{C}$ for 15 seconds and $62^{\circ} \mathrm{C}$ for 40 seconds. All reactions were run in triplicate. The sequence of specific BC200 RT primers was 5'-AGACCTGCCTGGGCAATATAGC-3', GAPDH was 5'-CATGAGAAGTATGACAACAGCCT-3'. The sequences of specific BC200 PCR primers: forward, 5'-AGACCTGCCTGGGCAATATAGC-3' and reverse, 5'-GTTGTTGCTTTGAGGGAAGTTACG-3'. The expression levels of BC200 were normalized to GAPDH (forward, 5'-CATGAGAAGTATGACAACAGCCT- $3^{\prime}$ and reverse, 5'-AGTCCTTCCACGATACCAAAGT-3'). Following the amplification, the same threshold was set for analyzing all experiments to compare $\mathrm{Ct}$ values, the mean $\mathrm{Ct}$ value was determined from the triplicate PCRs. The expression levels of BC200 were normalized to the internal control GAPDH reference to obtain the relative threshold cycle $(\triangle \mathrm{CT})$. We considered the mean $\mathrm{Ct}$ value of paired adjacent normal tissue as a standard control. The relative expression levels of cancer tissue were calculated by the comparative CT $\left({ }^{\Delta \Delta C T}\right)$ method, and relative expression folds $\left(2^{-\triangle \Delta \mathrm{CT}}\right)$ were calculated. And we grouped patients into high and low BC200 groups by a cut-off value determined based on the median cancer/adjacent tissue (control) ratio..$^{15}$

\section{Statistical analysis}

Comparisons of BC200 expression between ESCC tissue and adjacent normal tissue were assessed with independent $t$-test. Overall survival (OS) was calculated from the date of surgery to the date of death or the last follow-up. Diseasefree survival (DFS) was defined from the date of surgery to the date of local or distant recurrence or the date of the last follow-up. Patient survival rates were calculated using the Kaplan-Meier method, and statistically significant differences in survival were identified using the Cox proportional hazard regression model analysis. Chi-square test was used to estimate the correlation between clinical-pathological features with BC200 expression levels. Univariate and multivariate analysis was performed to identify the impact of clinical-pathological factors and BC200 expression level on
DFS and OS in ESCC patients who received radical resection. All $P$-values were two tailed. The significance levels of $P$-value were considered $<0.05$. All analyses were carried out using the SPSS software version 20.0 (IBM Corporation, Armonk, NY, USA) and GraphPad Prism 5.01 (GraphPad Software, Inc., La Jolla, CA, USA).

\section{Results \\ BC200 expression was increased in ESCC tissue}

We compared the expression level of $\mathrm{BC} 200$ between cancer and paired adjacent tissues. Our results showed that BC200 expression level was increased in $57.1 \%$ (40/70) of the tumor tissues, and was significantly higher than that of matched adjacent tissues $(P=0.023$, Figure 1$)$. We then grouped patients into high and low BC200 groups by a cut-off value of 1.39, determined based on the median cancer/control ratio.

\section{Correlation of BC200 expression with clinical-pathological characteristics}

Next we correlated BC200 expression levels with the clinical-pathological characteristics of patients with ESCC (Table 2). We failed to find the relative expression of BC200 that had any relation with other clinical-pathological characteristics such as patient age, $T$ stage, sex, lymph node status, TNM stage, histological grade, pathological type, and location (Table 2).

\section{High expression levels of $\mathrm{BC} 200$ are correlated with poor prognosis in ESCC patients}

To further evaluate whether BC200 upregulation was linked to the prognosis of patients who have received radical resection of ESCC, we analyzed the prognostic value of $\mathrm{BC} 200$ expression levels in 70 cancer patients using Kaplan-Meier

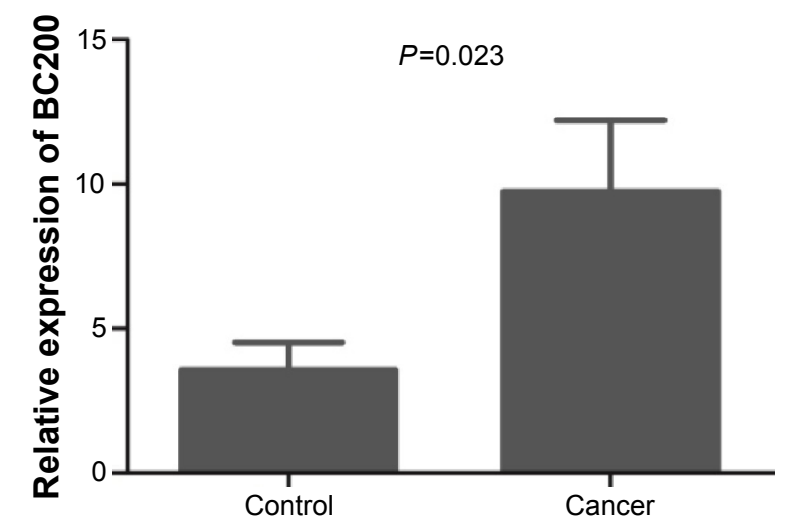

Figure I BC200 expression was increased in esophageal squamous cell carcinoma tissues compared to the adjacent normal tissues $(P=0.023)$. 
Table 2 The correlation of clinicopathological characteristics with BC200 expression

\begin{tabular}{|c|c|c|c|}
\hline \multirow[t]{2}{*}{ Characteristics } & \multicolumn{2}{|c|}{ BC200 Expression } & \multirow[t]{2}{*}{$P$-value } \\
\hline & Low N (\%) & High N (\%) & \\
\hline \multicolumn{4}{|l|}{ Sex } \\
\hline Male & $21(60.00)$ & 25 (7I.43) & 0.31 \\
\hline Female & $14(40.00)$ & $10(28.57)$ & \\
\hline \multicolumn{4}{|l|}{ Age (years) } \\
\hline$\leq 60$ & $13(37.14)$ & $8(22.86)$ & 0.19 \\
\hline$>60$ & $22(62.86)$ & $27(77.14)$ & \\
\hline \multicolumn{4}{|l|}{ pT } \\
\hline TI-2 & $7(20.00)$ & $8(22.86)$ & 0.77 \\
\hline T3-4 & $28(80.00)$ & $27(77.14)$ & \\
\hline \multicolumn{4}{|l|}{ pN } \\
\hline Negative & $19(54.29)$ & $19(54.29)$ & 1.00 \\
\hline Positive & $16(45.7 I)$ & $16(45.7 I)$ & \\
\hline \multicolumn{4}{|c|}{ Histological grade } \\
\hline $\mathrm{I}-2$ & $17(5 \mid .4)$ & $22(37.1)$ & 0.22 \\
\hline 3 & $18(48.6)$ & $13(62.9)$ & \\
\hline \multicolumn{4}{|l|}{ TNM stage } \\
\hline$|-| \mid$ & $21(60.00)$ & $24(68.58)$ & 0.45 \\
\hline III & $14(40.00)$ & II (3I.42) & \\
\hline \multicolumn{4}{|l|}{ Cancer location } \\
\hline Upper/middle & $26(74.29)$ & $23(65.7 \mathrm{I})$ & 1.00 \\
\hline Lower & $9(25.7 \mathrm{I})$ & $12(34.29)$ & \\
\hline
\end{tabular}

analysis and the log-rank test. The median follow-up period for the patients studied was 38 months. Among the 35 patients with high BC200 expression levels, 22 (62.86\%) developed local and/or distant recurrence, and 20 (57.14\%) died. However, 16 of 35 (45.71\%) with low BC200 expression levels developed local and/or distant recurrence, and $14(40.00 \%)$ patients died. Patients with a high level of BC200 had a shorter DFS than those with low BC200 expression $(P=0.034$, Figure 2). More importantly, the high BC200 expression group had shorter OS than the low expression group ( $P=0.031$, Figure 3$)$.

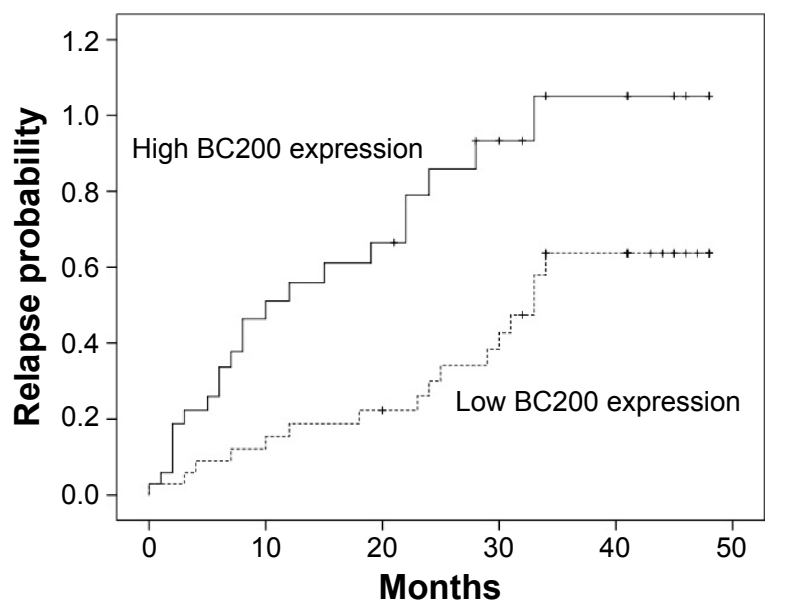

Figure 2 Kaplan-Meier curve of BC200 expression of patients with esophageal squamous cell carcinoma by disease-free survival $(P=0.034)$.

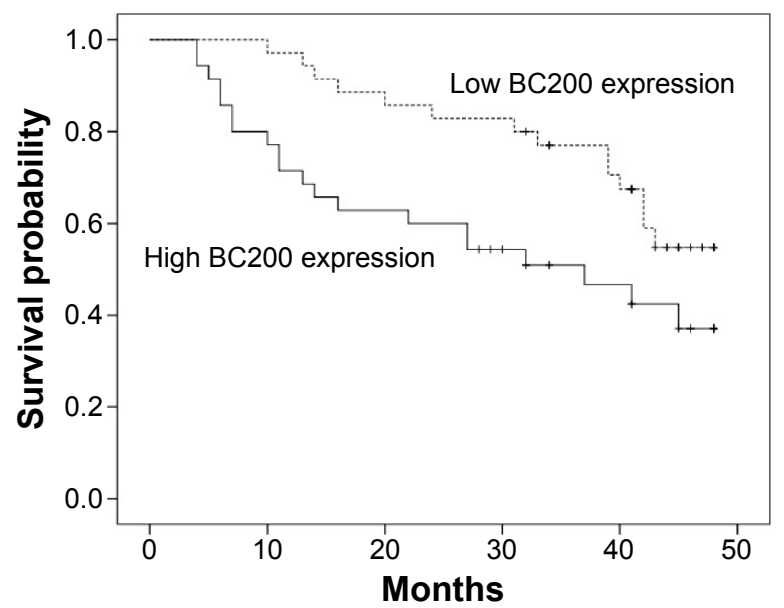

Figure 3 Kaplan-Meier curve of $B C 200$ expression of patients with esophageal squamous cell carcinoma by overall survival $(P=0.031)$.

Univariate analysis was used to analyze association of various conventional clinical-pathological factors and BC200 levels with the DFS and OS. Our results showed that only BC200 expression was associated with DFS and OS, other traditional factors, such as lymph node status and TNM stage, histological grade, adjuvant chemotherapy, and/or radiotherapy, had no significant correlation with DFS and OS (Table 3). As TNM stage was a definitive predictive factor for ESCC patients, we further analyzed BC200 expression and TNM stage with multivariate Cox regression model, adjusting the multivariate analysis by TNM stage. The BC200 expression was identified as independent prognostic factor of poor prognosis for DFS and OS (hazard ratio [HR] $=2.17,95 \%$ confidence interval $[\mathrm{CI}]=1.12-4.19, P=0.022$; and $\mathrm{HR}=2.24,95 \%$ $\mathrm{CI}=1.12-4.49, P=0.023$; Table 4).

\section{Discussion}

ESCC is a heterogeneous disease; prognosis of these patients might be totally different. So far, there are few reliable markers available to accurately predict metastasis in early stage ESCC patients, and individual adjuvant treatment remains a challenge. Therefore, more studies are needed for detection of reliable markers that are associated with advanced tumor progression in the early stage ESCC patients.

Recently, the roles for LncRNAs as tumor suppressive and oncogenic functions have been found in prevalent cancer types. Initially, BC200 was found expressed only in the primate nervous system, hence it was also called BCYRN1 (brain cytoplasmic RNA 1). The first study that linked BC200 to cancer was in $1997 .{ }^{14}$ In this study, 80 different tumor specimens were screened, representing 19 different tumor types; BC200 RNA was found to express in carcinomas of the 
Table 3 Univariate analysis of BC200 expression and clinical factors predictive of DFS and OS in 70 ESCC patients

\begin{tabular}{|c|c|c|c|c|}
\hline \multirow[t]{2}{*}{ Factors } & \multicolumn{2}{|l|}{ os } & \multicolumn{2}{|l|}{ DFS } \\
\hline & HR (95\% Cl) & $P$-value & HR (95\% Cl) & $P$-value \\
\hline BC200 & $2.08(1.05-4.14)$ & 0.036 & $1.98(1.03-3.79)$ & 0.039 \\
\hline Age & $1.26(0.59-2.70)$ & 0.556 & I.52 (0.72-3.22) & 0.272 \\
\hline Sex & $1.50(0.76-2.96)$ & 0.240 & I.I $9(0.62-2.28)$ & 0.605 \\
\hline Histological grade & $1.03(0.52-2.02)$ & 0.939 & $0.83(0.44-1.56)$ & 0.557 \\
\hline Adjuvant chemotherapy and/or radiotherapy & I.II (0.56-2.I7) & 0.767 & I.22 (0.64-2.32) & 0.543 \\
\hline pT & $1.37(0.57-3.32)$ & 0.481 & $1.38(0.6 \mathrm{I}-3.13)$ & 0.442 \\
\hline $\mathrm{pN}$ & $1.63(0.83-3.21)$ & 0.159 & $1.62(0.85-3.06)$ & 0.141 \\
\hline TNM stage & $1.60(0.8 I-3.14)$ & 0.177 & $1.60(0.84-3.06)$ & 0.152 \\
\hline Cancer location & $1.15(0.67-1.98)$ & 0.624 & I.2। (0.72-2.03) & $0.47 \mid$ \\
\hline
\end{tabular}

Note: Statistical significance, $P<0.05$.

Abbreviations: $\mathrm{Cl}$, confidence interval; DFS, disease-free survival; ESCC, esophageal squamous cell carcinoma; HR, hazard ratio; OS, overall survival.

breast, cervix, esophagus, lung, ovary, parotid, and tongue, but not in corresponding normal tissues. BC200 RNA was not detected in bladder, colon, kidney, or liver carcinoma tissues. Another study also found that BC200 RNA is expressed at high levels in invasive carcinomas of the breast. ${ }^{16}$ Recently, a study demonstrated that BC200 expression was significantly higher in non-small-cell lung cancer (NSCLC) tissue was than that in the adjacent normal tissue. The study also identified that c-MYC can regulate lung cancer cell migration and invasion via $\mathrm{BC} 200 .{ }^{17}$ However, they did not show the prognostic value of high levels of BC200 in NSCLC patients. There is no data to show that the expression level of BC200 in tumor tissues, especially in ESCC, could predict patient prognosis. Our results showed that BC200 expression was increased significantly in ESCC tissue than that in adjacent normal tissue samples $(P=0.023)$.

After analyzing the relationship between BC200 level in tumor tissues and clinical-pathological characteristics in ESCC patients, we failed to find the relationship between BC200 level and other clinical-pathological characteristics, such as patient age, $\mathrm{T}$ stage, sex, lymph node status, TNM stage, histological grade, pathological type, and location. Kaplan-Meier analysis showed that high expression levels of BC200 were correlated with poor prognosis in ESCC patients. Patients with a high level of BC200 had a shorter DFS and OS than those with low BC200 expression

Table 4 Multivariate analysis OS and DFS for the 70 ESCC patients

\begin{tabular}{|c|c|c|c|c|}
\hline \multirow[t]{2}{*}{ Factors } & \multicolumn{2}{|l|}{ os } & \multicolumn{2}{|l|}{ DFS } \\
\hline & HR (95\% Cl) & $P$-value & HR (95\% Cl) & $P$-value \\
\hline BC200 & $2.24(1.12-4.49)$ & 0.023 & $2.17(1.12-4.19)$ & 0.022 \\
\hline TNM stage & $1.77(0.90-3.52)$ & 0.100 & I.8I (0.94-3.50) & 0.076 \\
\hline
\end{tabular}

Note: Statistical significance, $P<0.05$.

Abbreviations: $\mathrm{Cl}$, confidence interval; DFS, disease-free survival; ESCC, esophageal squamous cell carcinoma; HR, hazard ratio; OS, overall survival.
$(P=0.022$ and 0.023 , respectively). Our results combined with other recent published studies revealed the important role of LncRNAs in ESCC cancer. For example, a recent study showed that MALAT-1 expressed higher levels in esophageal cancer tissues compared with paired adjacent normal tissues. This high expression was associated with a decreased survival rate. MALAT-1 knockdown induced a decrease in proliferation-enhanced apoptosis, inhibited migration/invasion, and reduced colony formation and led to cell cycle arrest at the G2/M phase. ${ }^{18}$ And there were some other LncRNA, such as CCAT2, PCAT1, UCA1, that were reported as prognosis marker for ESCC. ${ }^{12,19,20}$ These studies indicate that LncRNAs could be exploited for therapeutic benefit for ESCC.

In our multivariate Cox regression analysis, we only found that BC200 was an independent prognostic factor of poor prognosis for DFS and OS $(\mathrm{HR}=2.17,95 \% \mathrm{CI}=1.12-4.19$, $P=0.022$; and $\mathrm{HR}=2.24,95 \% \mathrm{CI}=1.12-4.49, P=0.023)$. This data showed that BC200 might play an important role in development and progression of ESCC. However, in our study other definitively reported factors, such as $\mathrm{T}$ stage, lymph node status, TNM stage, adjuvant chemotherapy, and/ or radiotherapy, were not identified as independent predictive markers for DFS and OS. ${ }^{1}$ That might be due to the following reasons: 1) The sample size of this study was too small, there were 70 patients in our study, in multivariate Cox regression analysis, $P$-value of TNM was $0.076(\mathrm{HR}=1.81$, $95 \% \mathrm{CI}=0.94-3.50)$ for $\mathrm{DFS}$ and $P=0.022(\mathrm{HR}=1.77,95 \%$ $\mathrm{CI}=0.90-3.52$ ) for $\mathrm{OS}$, which suggested that TNM might be a prospective factor for OS and DFS in ESCC patients, but need further investigation with more patients. 2) Patients selection: since it was a retrospective study, only ESCC patients who received radical resection were included in this study. Most of the patients were T3 or more (78.57\%), lymph node negative (64.94\%), and TNM stage I-II (64.28\%). Whether patients 
would receive adjuvant chemotherapy and/or radiotherapy depended mostly according to their financial situation and their wishes.

\section{Conclusion}

In conclusion, our analysis showed that BC200 expression was increased in ESCC tissues. Patients with a high level of BC200 had a shorter DFS and OS than those with low BC200 expression. Based on these results, BC200 expression may serve as a novel predictive marker for ESCC patients who received radical resection. Further investigation is warranted to understand more about the functions and mechanisms of $\mathrm{BC} 200$ in the development of ESCC.

\section{Acknowledgments}

We thank Dr Weijie Zhang and Prof Lijie Song for collecting parts of clinical data. We also thank Profs Changing Guo and Xiaobing Chen for giving advice on writing the article. This work was supported by a grant from The First Affiliated Hospital of Zhengzhou University for Young Scientists and by the National Natural Science Foundation of China (Grants 81472714/H1606). The funders had no role in data analysis, the decision to publish, or in preparation of the manuscript.

\section{Disclosure}

The authors report no conflict of interest in this work.

\section{References}

1. Napier KJ, Scheerer M, Misra S. Esophageal cancer: A review of epidemiology, pathogenesis, staging workup and treatment modalities. World J Gastrointest Oncol. 2014;6(5):112-120.

2. Sjoquist KM, Burmeister BH, Smithers BM, et al. Survival after neoadjuvant chemotherapy or chemoradiotherapy for resectable oesophageal carcinoma: an updated meta-analysis. Lancet Oncol. 2011;12(7): 681-692.

3. Gallo A, Cha C. Updates on esophageal and gastric cancers. World J Gastroenterol. 2006;12(20):3237-3242.
4. Ohashi S, Miyamoto S, Kikuchi O, Goto T, Amanuma Y, Muto M. Recent advances from basic and clinical studies of esophageal squamous cell carcinoma. Gastroenterology. 2015;149(7):1700-1715.

5. Mercer TR, Mattick JS. Structure and function of long noncoding RNAs in epigenetic regulation. Nat Struct Mol Biol. 2013;20(3):300-307.

6. Wang KC, Chang HY. Molecular mechanisms of long noncoding RNAs. Mol Cell. 2011;43(6):904-914.

7. Silva A, Bullock M, Calin G. The clinical relevance of long non-coding RNAs in cancer. Cancers (Basel). 2015;7(4):2169-2182.

8. Taylor DH, Chu ET, Spektor R, Soloway PD. Long non-coding RNA regulation of reproduction and development. Mol Reprod Dev. 2015; 82(12):932-956.

9. Chen FJ, Sun M, Li SQ, et al. Upregulation of the long non-coding RNA HOTAIR promotes esophageal squamous cell carcinoma metastasis and poor prognosis. Mol Carcinog. 2013;52(11):908-915.

10. Li X, Wu Z, Mei Q, Guo M, Fu X, Han W. Long non-coding RNA HOTAIR, a driver of malignancy, predicts negative prognosis and exhibits oncogenic activity in oesophageal squamous cell carcinoma. Br J Cancer. 2013;109(8):2266-2278.

11. Xie HW, Wu QQ, Zhu B, et al. Long noncoding RNA SPRY4-IT1 is upregulated in esophageal squamous cell carcinoma and associated with poor prognosis. Tumour Biol. 2014;35(8):12.

12. Li JY, Ma X, Zhang CB. Overexpression of long non-coding RNA UCA1 predicts a poor prognosis in patients with esophageal squamous cell carcinoma. Int J Clin Exp Pathol. 2014;7(11):7938-7944.

13. MusE,HofPR, TiedgeH.Dendritic BC200 RNA in aging and in Alzheimer's disease. Proc Natl Acad Sci U S A. 2007;104(25):10679-10684.

14. Chen W, Bocker W, Brosius J, Tiedge H. Expression of neural BC200 RNA in human tumours. J Pathol. 1997;183(3):345-351.

15. Zheng HT, Shi DB, Wang YW, et al. High expression of IncRNA MALAT1 suggests a biomarker of poor prognosis in colorectal cancer. Int J Clin Exp Pathol. 2014;7(6):3174-3181.

16. Iacoangeli A, Lin Y, Morley EJ, et al. BC200 RNA in invasive and preinvasive breast cancer. Carcinogenesis. 2004;25(11):2125-2133.

17. Hu T, Lu YR. BCYRN1, a c-MYC-activated long non-coding RNA, regulates cell metastasis of non-small-cell lung cancer. Cancer Cell Int. 2015;15:36.

18. Yao W, Bai Y, Li Y, et al. Upregulation of MALAT-1 and its association with survival rate and the effect on cell cycle and migration in patients with esophageal squamous cell carcinoma. Tumour Biol. Epub 2015, Oct 22 .

19. Wang J, Qiu M, Xu Y, et al. Long noncoding RNA CCAT2 correlates with smoking in esophageal squamous cell carcinoma. Tumour Biol. 2015;36(7):5523-5528.

20. Shi WH, Wu QQ, Li SQ, et al. Upregulation of the long noncoding RNA PCAT-1 correlates with advanced clinical stage and poor prognosis in esophageal squamous carcinoma. Tumour Biol. 2015;36(4): 2501-2507.
OncoTargets and Therapy

\section{Publish your work in this journal}

OncoTargets and Therapy is an international, peer-reviewed, open access journal focusing on the pathological basis of all cancers, potential targets for therapy and treatment protocols employed to improve the management of cancer patients. The journal also focuses on the impact of management programs and new therapeutic agents and protocols on

\section{Dovepress}

patient perspectives such as quality of life, adherence and satisfaction. The manuscript management system is completely online and includes a very quick and fair peer-review system, which is all easy to use. Visit http://www.dovepress.com/testimonials.php to read real quotes from published authors. 